\title{
Psychosocial Mediators between Socioeconomic Status and Dietary Restrictions among Patients Receiving Hemodialysis in Japan
}

\author{
Hidehiro Sugisawa ${ }^{D},{ }^{1}$ Toshio Shinoda, ${ }^{2}$ Yumiko Shimizu, ${ }^{3}$ \\ Tamaki Kumagai, ${ }^{4}$ and Hiroaki Sugisaki ${ }^{5}$ \\ ${ }^{1}$ Graduate School of Gerontology, J. F. Oberlin University, Machida-shi 194-0294, Japan \\ ${ }^{2}$ Faculty of Medical and Health Sciences, Tsukuba International University, Tsuchiura-city 300-0051, Japan \\ ${ }^{3}$ Faculty of Nursing, The Jikei University School of Medicine, Chofu-shi 182-8570, Japan \\ ${ }^{4}$ Graduate School of Nursing, Osaka City University, Osaka-shi 545-0051, Japan \\ ${ }^{5}$ Hachioji Azumacho Clinic, Hachioji-shi 192-0082, Japan \\ Correspondence should be addressed to Hidehiro Sugisawa; sugisawa@obirin.ac.jp
}

Received 3 December 2018; Accepted 31 March 2019; Published 17 April 2019

Academic Editor: Franca Anglani

Copyright (c) 2019 Hidehiro Sugisawa et al. This is an open access article distributed under the Creative Commons Attribution License, which permits unrestricted use, distribution, and reproduction in any medium, provided the original work is properly cited.

\begin{abstract}
The generalizability of differences in dietary restrictions (DRs) as function of socioeconomic status (SES) and the pathways of the associations between SES and DRs remain unclear. Therefore, we aimed to explore SES differences in DRs and psychosocial mediators between SES and DRs in Japanese patients receiving hemodialysis. This study was a cross-sectional survey of 6,644 outpatients (average age $=66.5$ years; $65 \%$ males) of hemodialysis facilities across Japan. DRs were assessed by self-reported and objective measures, and SES was assessed based on education and income. Three psychosocial mediators were used: self-efficacy, control expectancy, and social support. Indirect influences of SES through the mediators were evaluated with a multiple mediator model. Although higher education was significantly associated with higher self-reported DRs, higher income was significantly associated with lower self-reported DRs. Significant SES differences in objective DRs were not observed. The relationships between education and self-reported DRs and objective DRs were significantly mediated by self-efficacy and/or control expectancy. The influences of income were mediated by social support. It becomes possible to design interventions targeting modifiable psychosocial factors including self-efficacy, control expectancy, and social support in order to reduce SES inequalities in DRs.
\end{abstract}

\section{Introduction}

Socioeconomic status (SES) influences a wide range of health and health-related indicators in patients receiving dialysis. Studies using quality of life (QOL) indicators as outcome measures have shown strong relationships between higher SES and increased QOL scores [1-4]. Higher SES in patients receiving dialysis is related to mental and physical health indicators, such as lower depression [5-7], less frequent complications [5], and higher levels of activities of daily living [7] as compared to patients with a lower SES. In addition, lower SES in patients receiving dialysis is significantly related to higher mortality [8-15]. As several studies have reported that SES indicators are related to several health and healthrelated indicators, fluid and dietary restrictions (DRs), which are health-maintenance factors in patients receiving dialysis, may be influenced by SES. In fact, according to a recent review by Lambert and colleagues [16], SES impacted these restrictions in patients receiving dialysis. However, since there have been scant studies on SES differences in fluid/DRs, especially in oriental societies such as China and Japan, which have different cultural background from Western society, the generalizability of the previous findings remains unclear. Concurrently, the mechanisms underlying SES differences in health and fluid/DRs among patients receiving dialysis have rarely been pursued. 
Psychosocial mediators that explain the influence of SES on health and health-related behaviors have been explored in diverse patient groups, such as patients with coronary heart diseases [17], diabetes [18, 19], asthma or rhinitis [20-22], myocardial infarction [23], bacteremia [24], and patients who are chronically ill [25]. In addition, several previous studies have clarified significant influences of psychosocial factors on fluid/DRs in patients receiving dialysis, including selfefficacy [26-33], social support [29, 30, 34-37], perceived barriers/benefits [38-40], and locus of control [34].

Studies of the psychosocial mediators between SES and health behaviors noted that social support [41-46], selfefficacy $[42-44,46]$, and perceived control $[43,46]$ are in inconsistently distributed across SES. Although it remains unclear whether distributions of these psychosocial factors differ by SES in patients receiving dialysis, psychosocial factors may work as mediators to explain the relationships between SES and fluid/DRs in patients receiving dialysis. Consequently, we explored not only SES differences in DRs, but also psychosocial mediators between these indicators in Japanese patients receiving hemodialysis.

We used educational attainment and income as measures of SES. While education, income, and occupational status indicators are frequently used as SES indicators [47], we excluded occupational status because most of the patients receiving hemodialysis in Japan were women or older adults, both of which were nonworkers. According to a large-scale survey conducted in 2011 involving members of the Japan Association of Kidney Disease Patients, in which a third of the patients undergoing dialysis participated, the labor force participation rate of women as well as patients over 65 years old was $16.8 \%$ and $16.0 \%$, respectively [48].We used both education and income indicators to determine if they had distinct influences on DRs. Education appears to be a better index of psychosocial resources, such as the ability to manage social systems (e.g., navigate the healthcare system), effectively regulate health behaviors, accrue social support, and develop a sense of self-efficacy [49]. Income is related to the possession of material or immaterial resources, such as better housing, food, and resources for mastering stressful and demanding situations (e.g., seeking professional help); thus, a high income provides the opportunity for a health-promoting lifestyle [49]. If psychosocial resources such as social support and self-efficacy, which may be largely influenced by education, have an influence on DRs [50], we hypothesized that education would affect DRs through psychosocial mediators more than income would.

\section{Materials and Methods}

2.1. Data. We analyzed cross-sectional data collected in 2016 from all outpatients and their physicians across 118 dialysis facilities that were members of the Japan Association of Dialysis Physicians. The survey process comprised five steps. First, we requested all 923 members of the Association to participate in the survey. Second, we sent self-administrated questionnaires to the 118 dialysis facilities that agreed to participate. Third, medical staff distributed the questionnaires to all outpatients. Questionnaires included an explanation of participants' rights, privacy, and so on. Fourth, after patients completed the questionnaires, they sealed them in an envelope and handed them to their physicians (i.e., physicians could not see patients' responses). Lastly, physicians answered questionnaires regarding their patients and sent both sets of questionnaires to the survey secretariat.

Overall, we obtained questionnaires from 6,783 outpatients; however, the number of paired questionnairesresponded to by both patients and their physicians-was 6,644 , which were subsequently included in our analyses.

\subsection{Measures}

2.2.1. DRs. DRs were measured using both self-reported (SDRs) and objective (ODRs) measures. Shimizu and Sugisawa [51] developed an SDRs measure that included intake restrictions of three minerals: salt, phosphorus, and potassium. These three items were included in reliable and valid scales [52]. Although the original questionnaires included water restrictions, we excluded this restriction because we allowed beverages between meals. To measure restriction, we used a 4-point Likert scale: do almost all the time (4), do sometimes (3), do a few times (2), and almost never do (1). Regarding reliability, we conducted a principal component analysis for the three items using eigenvalues $>1.0$ criterion. Only a single component was extracted and all principal component scores of the three items exceeded 0.75 . The first component explained $68.8 \%$ of the total variance in the three items. Cronbach's alpha was .77. Regarding validity, we examined whether this measure could predict patients with the acceptable serum potassium range: lower than 6.0 $\mathrm{mEq} / \mathrm{l}$. Some studies showed that patients with $6.0 \mathrm{mEq} / \mathrm{l}$ and over had a significantly increased mortality risk $[53,54]$. We also examined whether this measure could discriminate patients with serum phosphorus of $3.5-5.5 \mathrm{mg} / \mathrm{dl}$. This range was recommended by the National Kidney foundation [55]. Maintaining serum phosphorus within this range may result in a better survival rate among patients receiving dialysis [56]. Consequently, SDRs significantly predicted an acceptable range of each medical indicator.

Levels of serum potassium and serum phosphorus were used to measure ODRs. We asked physicians to give the most recent numeric values of the two clinical indicators for each patient. As mentioned above, each criterion of acceptable DRs was lower than $6.0 \mathrm{mEq} / \mathrm{l}$ for serum potassium and 3.5-5.5 $\mathrm{mg} / \mathrm{dl}$ for serum phosphorus. In prior studies, Baraz et al. [57] defined dietary compliance as serum creatinine, sodium, serum potassium, calcium, phosphate, albumin, uric acid, and blood urea nitrogen being within the acceptable ranges, and Chan et al. [58] also considered dietary compliance as both serum potassium and phosphorus being within the acceptable ranges. They defined the lowest health risk group as dietary compliant using multiple medical indicators. By following their criteria, effective DRs were judged as having both serum potassium and phosphorus levels fall within the acceptable ranges in this study.

2.2.2. SES. SES was measured by educational attainment and household income. We asked participants to indicate 
their highest level of educational attainment: "junior high school," "high school," "vocational school," "junior college," "university," or "graduate school." We assigned 9, 12, 13, 14, 16 , and 18 to each category to quantify their responses. These figures reflect one's number of years of education according to the Japanese educational system. Regarding income, we calculated family-size adjusted income by dividing household annual income by the square root of household size. Eight income levels from "under one million yen (US \$8,900)" to "over ten million yen" (US $\$ 89,000$ ) were used to assess household annual income. We assigned midpoints to each income category to quantify the responses.

2.2.3. Possible Psychosocial Mediators for DRs. We originally created three scales to measure possible psychosocial mediators: self-efficacy, control expectancy, and social support, which were based on scales used in past research that explored psychosocial mediators between SES and dietary habits in older adults [46]. Content validity for each item was confirmed by three dialysis physicians and two patients receiving hemodialysis.

Self-Efficacy. Participants were asked questions about their confidence levels concerning whether they could not consume food in each of the following three scenarios: (a) when their favorite foods were right in front of them, (b) when they were very hungry, and (c) when others recommend the foods. Responses were scored using a four-point scale $(1=$ not at all confident to $4=$ very confident). Responses were summed to provide one self-efficacy score. A principal component analysis extracted a single component by imposing an eigenvalue > 1.0 criterion, which explained $78.9 \%$ of the total variance. All principal component scores exceeded .88. Cronbach's alpha of this index was 0.88 .

Control Expectancy. Regarding health maintenance, participants were asked to indicate the importance of (a) restricting their salt intake, (b) not eating foods high in potassium, and (c) not eating foods high in phosphorus. Responses were scored using a four-point scale $(1=$ not important to $4=$ very important). Responses were summed to provide one control expectancy score. A principal component analysis extracted a single component by imposing an eigenvalue $>1.0$ criterion, which explained $74.5 \%$ of the total variance. All principal component scores exceeded 0.80 . Cronbach's alpha of this index was 0.84 .

Social Support. Participants were asked about the level of perceived social support they received from their family/friends in each of the following three scenarios: (a) family/friends understand how hard it is to perform DRs, (b) family/friends cooperate with participants' performance, and (c) family/friends offer advice concerning DRs. Responses were scored using a four-point scale ( 1 = not supportive/no support to $4=$ very supportive). Responses were summed to provide one social support score. A principal component analysis extracted a single component by imposing an eigenvalue > 1.0 criterion, which explained $79.7 \%$ of the total variance. All principal component scores exceeded 0.88 . Cronbach's alpha for this index was 0.87 .

2.2.4. Control Variables. Sex $(1=$ male, $0=$ female $)$, age, period of dialysis, and activities of daily living were included as control variables. Period of dialysis (years) was from the initiation of dialysis to survey commencement. Activities of daily living were measured by levels of support needed to perform five activities: (1) ambulation indoors, (2) change clothes, (3) eat a meal, (4) take a bath, and (5) use a toilet. Responses ranged from "do at his/her ease" to "always need support to do it as he/she cannot do it." Participants who chose "do at his/her ease" for all five activities were assigned zero points. Participants who chose "have a little difficulty with $i t$ " or responses with more severe difficulties for at least one activity were assigned one point. Preliminary analysis showed that neither SDRs nor ODRs were influenced by primary causes of kidney diseases and adding these variables to the analytic model decreased model fitness. Consequently, we excluded these variables from analyses.

2.3. Statistical Analyses. We conducted multiple mediation analyses as proposed by Preacher and Hayes $[59,60]$ to determine the total and specific indirect effects in a multiple factor model. Analyses were conducted with Mplus Version 8.1 software; Muthén \& Muthén, Los Angeles, CA, USA [61]. In addition, we examined the null hypotheses that the indirect effects of each mediator resulting from the education and income indicators were equal [61]. We standardized all variables in the model to compare the sizes of the indirect effects produced by each mediator. We used bootstrapping (the number of bootstrap samples $=5,000$ ) to estimate the total and specific indirect effects of the mediators. We determined the point estimates and $95 \%$ confidence intervals per the null hypothesis.

We employed a full information maximum likelihood approach to handle missing data in the analysis [61]. We examined whether there were mediation effects, even if the total effects of education and income did not have significant effects on SDRs and ODRs. This approach was based on the suggestion that the significant effect of the independent variable is not always necessary for mediation to occur [59]. For example, if $\mathrm{M}_{1}$ acts as a mediator and a second mediator, $M_{2}$, acts as a suppressor, the total effects of the independent variable on the dependent variable might be reduced, given the possibility that the indirect effects of $\mathrm{M}_{1}$ and $\mathrm{M}_{2}$ cancel each other out. We assessed the overall model fit using the root mean square error of approximation (RMSEA) and the comparative fit index (CFI). It is recommended that the RMSEA be below 0.05 [62], and the CFI be above 0.90 [63].

2.4. Ethical Considerations. This study was conducted per the guidelines of the Helsinki Declaration, and all procedures were approved by the Research Ethics Board of J. F. Oberlin University. The questionnaire, along with the letter of invitation explaining the study content, was given to each potential survey respondent. Data collection procedures assured confidentiality using self-administered, anonymous questionnaires. Responses were completely voluntary, and 
TABLE 1: Descriptive statistics and correlations among study variables.

\begin{tabular}{|c|c|c|c|c|c|c|c|c|c|c|c|}
\hline Variable & Mean & SD & 1 & 2 & 3 & 4 & 5 & 6 & 7 & 8 & 9 \\
\hline 1. Age & 66.45 & 11.68 & - & & & & & & & & \\
\hline 2. Sex & 0.65 & 0.48 & -.006 & & & & & & & & \\
\hline 3. Duration of dialysis & 10.36 & 9.38 & $-.081^{* * *}$ & $-.098^{* * *}$ & & & & & & & \\
\hline 4. Disabled ADL & 0.3 & 0.46 & $.286^{* * *}$ & $-.067^{* * *}$ & $.044^{* * *}$ & & & & & & \\
\hline 5. Education & 18.35 & 2.38 & $-.257^{* * *}$ & $.157^{* * *}$ & $.054^{* * *}$ & $-.153^{* * *}$ & & & & & \\
\hline 6. Income & 2.41 & 1.64 & $-.098^{* * *}$ & $.041^{* * *}$ & .004 & $-.136^{* * *}$ & $.310^{* * *}$ & & & & \\
\hline 7. SE & 7.90 & 1.89 & $.126^{* * *}$ & $-.027^{*}$ & $.027^{*}$ & -.009 & .001 & -.019 & & & \\
\hline 8. CE & 9.67 & 1.58 & .000 & $-.081^{* * *}$ & $-.041^{* * *}$ & $-.036^{* *}$ & .024 & $.026^{*}$ & $.342^{* * *}$ & & \\
\hline 9. SS & 8.95 & 1.92 & $.236^{* * *}$ & $.031^{*}$ & $-.080^{* * *}$ & $.054^{* * *}$ & $-.030^{*}$ & $.046^{* * *}$ & $.261^{* * *}$ & $.258^{* * *}$ & \\
\hline 10. Self-reported DRs & 8.81 & 1.56 & $.167^{* * *}$ & $-.078^{* * *}$ & $-.063^{* * *}$ & $.061^{* * *}$ & $-.041^{* * *}$ & $-.063^{* * *}$ & $.455^{* * *}$ & $.395^{* * *}$ & $.316^{* * *}$ \\
\hline 11. Objective DRs & 0.63 & - & - & - & - & - & - & - & - & - & - \\
\hline
\end{tabular}

Note 1: SD: standard deviation, ADL: activities of daily living, SE: self-efficacy for dietary restrictions, CE: control expectancy for dietary restrictions, SS: social support for dietary restrictions, and DRs: dietary restrictions.

Note 2: means and correlations were calculated using the full-information maximum likelihood method.

Note 3: means and standard deviations represent values before the variables were standardized.

Note 4: objective dietary restrictions represent a dependent and categorical variable; we did not calculate its correlations with the independent variables.

Note $5:{ }^{*} P<.05,{ }^{* *} P<.01$, and ${ }^{* * *} P<.001$.

confidentiality was fully guaranteed. As we asked participants to return the completed questionnaires only if they agreed to participate in the survey, only respondents who wished to participate returned their questionnaires, thus implying consent.

\section{Results}

3.1. Descriptive Statistics. Table 1 shows participants' descriptive statistics and correlations among study variables. Sex, dialysis duration, and disabled activities of daily living were control variables. Multicollinearity was not a concern. Regarding SDRs, the path coefficients of education and income were significant after considering age, sex, activities of daily living, and other SES indicators $(0.035, P=.013$; $-0.056, P<.001$, respectively). Regarding ODRs, the path coefficients of education and income were nonsignificant after considering age, sex, activities of daily living, and other SES indicators $(\mathrm{B}=-0.020, P=.277 ; \mathrm{B}=-0.018, P=.337$, respectively).

3.2. Multiple Mediation. Figure 1 displays the results of the multiple mediation analysis regarding SDRs. The model fit was acceptable. Most direct effects of education on selfefficacy and control expectancy as mediators of SDRs were significant. However, education had no significant direct effects on support. On the other hand, the direct effects of income only on social support were significant. Each direct effect of self-efficacy, control expectancy, and social support on SDRs was significant. Multiple mediation analyses showed that self-efficacy and control expectancy were significant mediators between education and SDRs. In addition, selfefficacy was a more significant mediator than was social support. After entering the three mediators in the analytic model, the effect of education on SDRs became smaller and nonsignificant $(P=.374)$. Only social support was a significant mediator between income and SDRs; however, income did not have significant total indirect effects on SDRs. Social support was a more significant mediator than was selfefficacy. After entering the three mediators in the analytic model, the direct negative effect of income on SDRs became larger by subtracting the positive indirect effects of income $(P$ $<.001)$.

Figure 2 provides the results of the multiple mediation analysis regarding ODRs. The model fit was moderately acceptable. The direct effects of both education and income on the three mediators were almost the same as the results for SDRs above. Each direct effect of self-efficacy and social support on ODRs was significant. Multiple mediation analyses showed that self-efficacy significantly mediated education and ODRs. In addition, self-efficacy was a more significant mediator than was control expectancy. After entering the three mediators in the analytic model, the direct negative effect of education on ODRs became larger by subtracting the positive indirect effects of education $(P=.192)$. Regarding the effects of income, only social support was a significant mediator between income and ODRs. Social support was a more significant mediator than was self-efficacy and control expectancy. After entering the three mediators in the analytic model, the direct negative effect of income on ODRs became larger by subtracting the positive indirect effects of income $(P$ $=.276)$.

\section{Discussion}

We hypothesized that SES differences are linked with DRs in patients receiving hemodialysis. In this study, higher education significantly influenced higher SDRs after considering the influence of control variables. This result supports our hypothesis. On the other hand, income had a significant influence on SDRs after considering the influence of the control variables, displaying a significant link between higher 


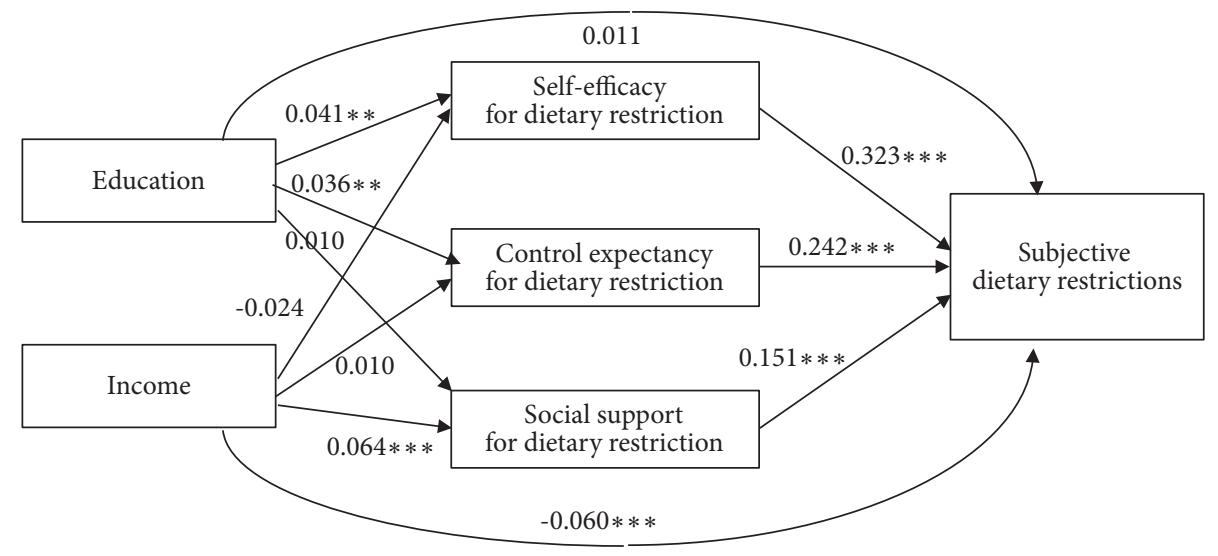

FIGURE 1: Direct and indirect effects of socioeconomic status on subjective dietary restrictions as a function of psychosocial mediators. Note 1: the effects of each variable were obtained after controlling for the influence of other variables without the variable in question. Note $2:{ }^{*} P<$ $.05,{ }^{* *} P<.01$, and ${ }^{* * *} P<.001$. Note 3 : total indirect influence of education on subjective dietary restrictions (SDRs) through three mediators was 0.024 (95\% confidence interval: 0.009 to 0.038 ); each indirect influence of education on SDRs through self-efficacy, control expectancy, and social support was 0.013 ( 0.004 to 0.023$), 00.009$ (0.002 to 0.014$)$, and $0.002(-0.002$ to 0.006$)$ separately. Note 4 : total indirect influence of income on SDRs through three mediators was 0.004 (95\% confidence interval:- 0.011 to 0.019 ); each indirect influence of income on SDRs through self-efficacy, control expectancy, and social support was -0.008 (-0.017 to 0.001$), 0.002$ (-0.005 to 0.009 ), and 0.010 (0.005 to 0.015 ) separately. Note 5: difference in indirect influences of education on SDRs through self-efficacy and control expectancy was 0.004 (-0.005 to 0.014 ) through self-efficacy and social support was 0.011 (0.003 to 0.021$)$; and through control expectancy and social support was 0.007 $(0.000$ to 0.014$)$. Note 6 : difference in indirect influences of income on SDRs through self-efficacy and control expectancy was 0.010 ( 0.000 to 0.020$)$; through self-efficacy and social support was 0.018 (0.008 to 0.027); difference in indirect influences of income on SDRs through control expectancy and social support was 0.008 (0.000 to 0.015$)$. Note 7: root mean square error of approximation $=0.077$; comparative fit index $=0.987$.

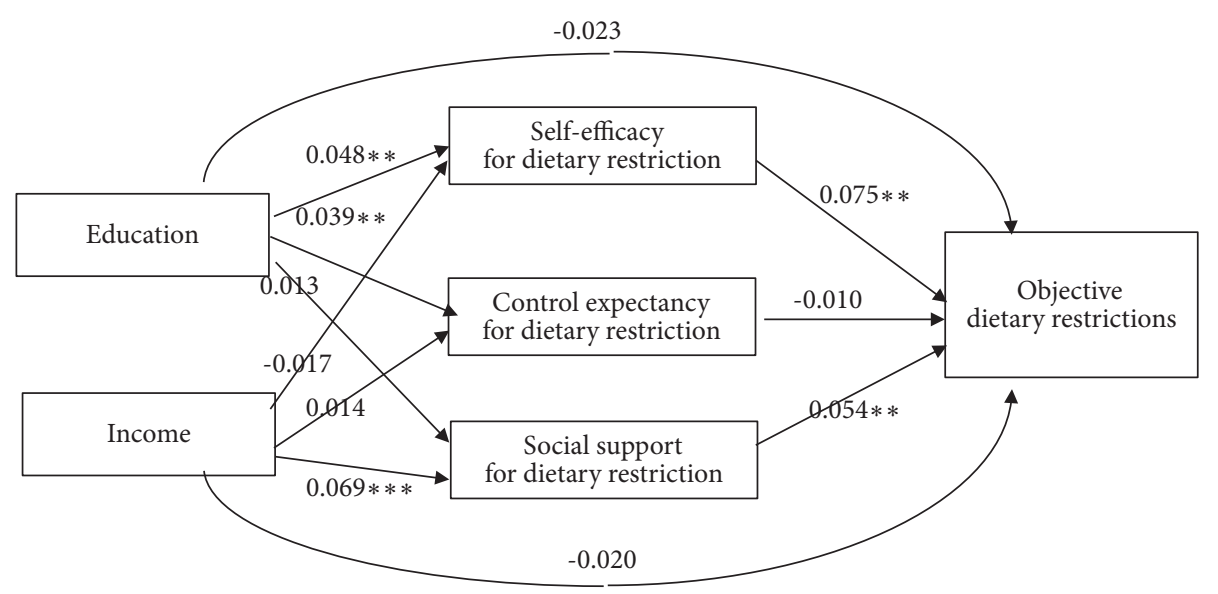

FIGURE 2: Direct and indirect effects of socioeconomic status on objective dietary restrictions as a function of psychosocial mediators. Note 1: the effects of each variable were obtained after controlling for the influence of other variables without the variable in question. Note $2:{ }^{*} P<$ $.05,{ }^{* *} P<.01$, and ${ }^{* * *} P<.001$. Note 3 : total indirect influence of education on objective dietary restrictions (ODRs) through three mediators was 0.004 (95\% confidence interval: 0.001 to 0.008 ); each indirect influence of education on ODRs through self-efficacy, control expectancy, and social support was 0.004 (0.001 to 0.007$), 0.000(-0.002$ to 0.001$)$, and $0.001(-0.001$ to 0.003$)$ separately. Note 4 : total indirect influence of income on ODRs through three mediators was 0.002 ( $95 \%$ confidence interval:- 0.001 to 0.006 ); each indirect influence of income on ODSs through self-efficacy, control expectancy, and social support was -0.001 (-0.004 to 0.001$), 0.000$ (-0.001 to 0.000$)$, and 0.004 (0.001 to 0.007 ) separately. Note 5: difference in indirect influences of education on ODRs through self-efficacy and control expectancy was 0.004 (0.001 to 0.008 ); through self-efficacy and social support was 0.003 ( 0.000 to 0.006 ); and through control expectancy and social support was 0.001 $(-0.001$ to 0.004$)$. Note 6 : difference in indirect influences of income on ODRs through self-efficacy and control expectancy was 0.001 ( -0.001 to 0.004$)$; through self-efficacy and social support was 0.005 (0.002 to 0.009); and through control expectancy and social support was 0.004 (0.001 to 0.008$)$. Note 7 : root mean square error of approximation $=0.080$; comparative fit index $=0.979$. 
income and lower SDRs, which contrasts our hypothesis. Some studies indicated that higher SES had significant negative influences on DRs $[36,64]$, while others showed significant links between higher income and higher DRs [16]. However, these authors did not explain the reasons for their results and noted the limited generalizability of their findings. Our current results are valid because of the large sample size, which was drawn from all outpatients receiving dialysis in Japan.

Why was higher income associated with lower SDRs? In this study, DR elements included phosphorus, potassium, and salts. In the general population, consumption of fruits and vegetables including consuming a lot of potassium is recommended; however, this differs for patients receiving dialysis [65]. In addition, protein-rich foods-including meat and fish-are recommended for older adults to have a healthy diet $[66,67]$. Protein-rich foods contain a lot of phosphorus and potassium, although additives are increasingly being added to processed and fast foods, particularly meat, cheese, baked goods, and beverages. A review article indicated that adults with a lower SES were less likely to consume fruit and vegetables $[64,68]$ and being older and having a lower individual and household income predicted a decreased consumption of fruits and vegetables [69-71]. Low-income adults also consume less meat and fish than do their wealthier counterparts [71]. One explanation for these findings is that fresh fruit and vegetables are expensive [72] and income is related to the possession of material or immaterial resources, such as better housing, food, and resources for dealing with stressful and demanding situations (e.g., seeking professional help) [49]. According to these findings in the general population, results of this study indicate that it is difficult for most higher income patients receiving dialysis to change their dietary habits because they were more likely to consume potassium- and phosphorus-rich foods before they had started receiving dialysis.

One reason for the nonsignificant SES differences in ODRs might be related to taking potassium- and phosphorus-lowering medication. According to systematic review, very few studies found low education to be significantly associated with medication nonadherence [73]. Therefore, the consistency of taking this medication across SES levels may contribute to the nonsignificant differences seen in patients receiving dialysis.

We further showed that SES indicators had indirect significant influences on both SDRs and ODRs through psychosocial mediators. Our findings suggest that the mechanisms for these are distinct depending on the SES indicator, education or income. Notably, education, income, and occupational status cannot be used interchangeably in social epidemiology [47]. In fact, some studies showed that each indicator predicted distinct health indicators $[47,74,75]$. However, these past studies did not determine the relevant mechanisms behind these differences. Regarding SES differences in mediational influence, it has been suggested that education can reflect a range of noneconomic psychosocial resources effectively to regulate health behaviors, accrue social support, and develop a sense of personal control or agency [49]. Further, income is a more direct index of material resources than is education, as it reflects one's ability to afford better healthcare, nutrition, housing, and so on, thus promoting better health and recovery [49].

According to this study, education had significant influences on SDRs and ODRs through self-efficacy and/or control expectancy, and income only had significant influences on SDRs and ODRs through social support. These results support the hypothesis that education influences DRs through boosting noneconomic psychosocial recourses such as selfefficacy. However, income was also mediated by social support, although the significant mediators differed from those for education. Why did social support mediate income and both indicators of DRs? Regarding support in general, it is hypothesized that the relationship between income and social support is mediated by life events, which disrupt and impair social relationships [76]. Among people with a lower income, social network members tend to share resources (albeit few) to enhance the person's capacity to cope with the problem. Patients receiving dialysis who belong to a lower income group are likely to have experienced negative life events owing to their limited resources. Consequently, approaching their networks with multiple events may overwhelm the network's limited recourses and availability. This may be the reason for the reduced influence of social support among low-income patients receiving dialysis.

Our study may have important public health and policy implications. By understanding the mediating factors that explain the relationship between SES and DRs, it becomes possible to design interventions targeting modifiable psychosocial factors, including self-efficacy, control expectancy, and social support, in order to reduce SES inequalities in DRs. The effect of these interventions may be most beneficial for lower SES patients receiving hemodialysis.

This study had some limitations. First, although we used a large, representative sample, the income differences in SDRs contrasted our hypothesis and prior results; therefore, future studies should attempt to validate our results including examining non-Japanese patients receiving dialysis. Second, we did not determine whether participants were taking medication to decrease their phosphorus or potassium levels, which could have affected the results. Third, although we included both self-reported and objective measures to evaluate DRs, other adherence measures-such as fluid restrictions, taking medicine, and the frequency of skipping dialysis - should be examined to determine if they are associated with patients' SES. Fourth, social desirability bias (i.e., the defensive tendency to present oneself in a more favorable light) might exist in responses regarding SDRs. A study reported that there social desirability bias exists in dietary self-report questionnaires [77]. Although this study did not include social desirability in the analytic framework, there is a need to identify social desirability bias using social desirability scales.

\section{Conclusions}

The results obtained from this study cannot provide a definitive answer to whether there are SES differences in DRs in Japanese patients receiving hemodialysis. However, despite its limitations, this study provided novel findings; specifically, 
although patients with low levels of education who receive hemodialysis may have lower SDRs than patients with a higher level of education, it is possible that higher income patients receiving hemodialysis have lower DRs than lower income patients. Further, although the influences of SES may be mediated through self-efficacy, control expectancy, and/or social support, these mediators may differ between education and income indicators.

\section{Data Availability}

The data are available from the corresponding author upon reasonable request.

\section{Conflicts of Interest}

The authors declare that there are no conflicts of interest regarding the publication of this paper.

\section{Acknowledgments}

We acknowledge the help of the many dialysis physicians from the Japan Association of Dialysis Physicians and the many outpatients at dialysis facilities who collaborated with this research. This work was conducted by research expenses of the Japan Association of Dialysis Physicians and the Institute of Statistical Research.

\section{References}

[1] C.-K. Chiang, Y.-S. Peng, S.-S. Chiang et al., "Health-related quality of life of hemodialysis patients in Taiwan: A multicenter study," Blood Purification, vol. 22, no. 6, pp. 490-498, 2004.

[2] M. C. Cruz, C. Andrade, M. Urrutia, S. Draibe, L. A. NogueiraMartins, and R. D. C. C. Sesso, "Quality of life in patients with chronic kidney disease," Clinics, vol. 66, no. 6, pp. 991-995, 2011.

[3] P. Rebollo, F. Ortega, J. M. Baltar et al., "Health-related quality of life (HRQOL) in end stage renal disease (ESRD) patients over 65 years.", Geriatric Nephrology and Urology, vol. 8, no. 2, pp. 85-94, 1998.

[4] R. Sesso, J. F. Rodrigues-Neto, and M. B. Ferraz, "Impact of socioeconomic status on the quality of life of ESRD patients," American Journal of Kidney Diseases, vol. 41, no. 1, pp. 186-195, 2003.

[5] H. Sugisawa, Y. Shimizu, T. Kumagai, H. Sugisaki, S. Ohira, and T. Shinoda, "Effects of socioeconomic status on physical and mental health of hemodialysis patients in Japan: Differences by age, period, and cohort," International Journal of Nephrology and Renovascular Disease, vol. 9, pp. 171-182, 2016.

[6] P. Theofilou, "Depression and anxiety in patients with chronic renal failure: the effect of sociodemographic characteristics," International Journal of Nephrology, vol. 2011, Article ID 514070, 6 pages, 2011.

[7] W. L. Cook and S. V. Jassal, "Functional dependencies among the elderly on hemodialysis," Kidney International, vol. 73, no. 11, pp. 1289-1295, 2008.

[8] A. Georgi, T. Jayaseelan, M. Milly et al., "Resource settings have a major influence on the outcome of maintenance hemodialysis patients in South India," Hemodialysis International, vol. 14, no. 2, pp. 211-217, 2010.
[9] F. J. Caskey, P. Roderick, R. Steenkamp et al., "Social deprivation and survival on renal replacement therapy in England and Wales," Kidney International, vol. 70, no. 12, pp. 2134-2140, 2006.

[10] P. L. Kimmel, C.-W. Fwu, and P. W. Eggers, "Segregation, income disparities, and survival in hemodialysis patients," Journal of the American Society of Nephrology, vol. 24, no. 2, pp. 293-301, 2013.

[11] S. Marinovich, C. Lavorato, G. Rosa-Diez, L. Bisigniano, V. Fernández, and D. Hansen-Krogh, "The lack of income is associated with reduced survival in chronic haemodialysis," Nefrología, vol. 32, no. 1, pp. 79-88, 2012.

[12] F. K. Port, R. A. Wolfe, N. W. Leven, K. E. Guire, and C. W. Ferguson, "Income and survival in chronic dialysis patients," American Society for Artificial Internal Organs Transactions, vol. 36, no. 3, pp. M154-M157, 1990.

[13] M. Sanabria, J. Mũoz, C. Trillos et al., "Dialysis outcomes in Colombia (DOC) study: A comparison of patient survival on peritoneal dialysis vs hemodialysis in Colombia," Kidney International, vol. 73, no. 108, pp. S165-S172, 2008.

[14] F. L. Ward, P. O'Kelly, F. Donohue et al., "The influence of socioeconomic status on patient survival on chronic dialysis," Hemodialysis International, vol. 19, no. 4, pp. 601-608, 2015.

[15] R. Xu, Q. Han, T. Zhu et al., "Impact of individual and environmental socioeconomic status on peritoneal dialysis outcomes: a retrospective multicenter cohort study," PLoS ONE, vol. 7, no. 11, Article ID e50766, 2012.

[16] K. Lambert, J. Mullan, and K. Mansfield, "An integrative review of the methodology and findings regarding dietary adherence in end stage kidney disease," BMC Nephrology, vol. 18, no. 1, article no 318, 2017.

[17] G. Barbareschi, R. Sanderman, G. I. J. M. Kempen, and A. V. Ranchor, "The mediating role of perceived control on the relationship between socioeconomic status and functional changes in older patients with coronary heart disease," Journals of Gerontology - Series B Psychological Sciences and Social Sciences, vol. 63, no. 6, pp. P353-P361, 2008.

[18] J. Houle, F. Lauzier-Jobin, M. D. Beaulieu et al., "Socioeconomic status and glycemic control in adult patients with diabetes a mediation analysis," BMJ Open Diabetes Research \& Care, vol. 4, no. 1, Article ID e000184, 2016.

[19] D. Schillinger, L. R. Barton, A. J. Karter, F. Wang, and N. Adler, "Does literacy mediate the relationship between education and health outcomes? A study of a low-income population with diabetes," Public Health Reports, vol. 121, no. 3, pp. 245-254, 2006.

[20] L. Trupin, P. P. Katz, J. R. Balmes et al., "Mediators of the socioeconomic gradient in outcomes of adult asthma and rhinitis," American Journal of Public Health, vol. 103, no. 2, pp. e31-e38, 2013.

[21] E. Chen, E. B. Fisher, L. B. Bacharier, and R. C. Strunk, "Socioeconomic status, stress, and immune markers in adolescents with asthma," Psychosomatic Medicine, vol. 65, no. 6, pp. 984-992, 2003.

[22] E. Chen, M. D. Hanson, L. Q. Paterson, M. J. Griffin, H. A. Walker, and G. E. Miller, "Socioeconomic status and inflammatory processes in childhood asthma: The role of psychological stress," The Journal of Allergy and Clinical Immunology, vol. 117, no. 5, pp. 1014-1020, 2006.

[23] K. K. Bennett, D. M. Buchanan, P. G. Jones, and J. A. Spertus, "Socioeconomic status, cognitive-emotional factors, and health status following myocardial infarction: testing the Reserve Capacity Model," Journal of Behavioral Medicine, vol. 38, no. 1, pp. 110-121, 2014. 
[24] K. Koch, M. Nørgaard, H. C. Schønheyder, R. W. Thomsen, M. Søgaard, and Danish Collaborative Bacteremia Network, "Effect of socioeconomic status on mortality after bacteremia in working-age patients: a Danish population-based cohort study," PLoS ONE, vol. 8, no. 7, Article ID e70082, 2013.

[25] T. Jansen, J. Rademakers, G. Waverijn, R. Verheij, R. Osborne, and M. Heijmans, "The role of health literacy in explaining the association between educational attainment and the use of out-of-hours primary care services in chronically ill people: A survey study," BMC Health Services Research, vol. 18, no. 1, p. 394, 2018.

[26] B. A. Brady, C. M. Tucker, P. A. Alfino, D. G. Tarrant, and G. C. Finlayson, "An investigation of factors associated with fluid adherence among hemodialysis patients: A self-efficacy theory based approach," Annals of Behavioral Medicine, vol. 19, no. 4, pp. 339-343, 1997.

[27] E. L. Lev and S. V. Owen, "A prospective study of adjustment to hemodialysis," American Nephrology Nurse's Association Journal, vol. 25, no. 5, pp. 495-504, 1998.

[28] M. Lindberg and M. A. M. Fernandes, "Self-efficacy in relation to limited fluid intake amongst Portuguese heamodialysis patients," Journal of Renal Care, vol. 36, no. 3, pp. 133-138, 2010.

[29] M. Oka, S. Tomura, H. Takahashi, and S. Tsuchiya, "Treatment regimen adherence and life satisfaction in hemodialysis patients: a covariance structure analysis," Clinical and Experimental Nephrology, vol. 3, no. 3, pp. 198-206, 1999.

[30] M. Oka and W. Chaboyer, "Influences of self-efficacy and other factors on dietary behaviours in Japanese hemodialysis patients," International Journal of Nursing Practice, vol. 7, no. 6, pp. 431-439, 2001.

[31] M. Rosenbaum and K. B.-A. Smira, "Cognitive and personality factors in the delay of gratification of hemodialysis patients," Journal of Personality and Social Psychology, vol. 51, no. 2, pp. 357-364, 1986.

[32] M. S. Schneider, R. Friend, P. Whitaker, and N. K. Wadhwa, "Fluid noncompliance and symptomatology in end-stage renal diseases: cognitive and emotional variables," Health Psychology, vol. 10, no. 3, pp. 209-215, 1991.

[33] M. Zrinyi, M. Juhasz, J. Balla et al., "Dietary self-efficacy: determinant of compliance behaviours and biochemical outcomes in haemodialysis patients," Nephrology Dialysis Transplantation, vol. 18, no. 9, pp. 1869-1873, 2003.

[34] J. Brown and R. Fitzpatrick, "Factors influencing compliance with dietary restrictions in dialysis patients," Journal of Psychosomatic Research, vol. 32, no. 2, pp. 191-196, 1988.

[35] B. Kara, K. Caglar, and S. Kilic, "Nonadherence with diet and fluid restrictions and perceived social support in patients receiving hemodialysis," Journal of Nursing Scholarship, vol. 39, no. 3, pp. 243-248, 2007.

[36] S.-K. Pang, W.-Y. Ip, and A. M. Chang, "Psychosocial correlates of fluid compliance among Chinese haemodialysis patients," Journal of Advanced Nursing, vol. 35, no. 5, pp. 691-698, 2001.

[37] M. Oka and W. Chaboyer, "Dietary behaviors and sources of support in hemodialysis patients," Clinical Nursing Research, vol. 8, no. 4, pp. 302-317, 1999.

[38] S.-H. Lee and A. Molassiotis, "Dietary and fluid compliance in Chinese hemodialysis patients," International Journal of Nursing Studies, vol. 39, no. 7, pp. 695-704, 2002.

[39] L. K. Thomas, R. G. Sargent, P. C. Michels, D. L. Richter, R. F. Valois, and C. G. Moore, "Identification of the factors associated with compliance to therapeutic diets in older adults with end stage renal disease," Journal of Renal Nutrition, vol. 11, no. 2, pp. 80-89, 2001.

[40] R. D. F. Agondi, M.-C. B. J. Gallani, R. C. M. Rodrigues, and M. E. Cornélio, "Relationship between beliefs regarding a low salt diet in chronic renal failure parents on dialysis," Journal of Renal Nutrition, vol. 21, no. 2, pp. 160-168, 2011.

[41] M. Droomers, C. T. M. Schrijvers, H. Van De Mheen, and J. P. Mackenbach, "Education differences in leisure-time physical inactivity: a descriptive and explanatory study," Social Science \& Medicine, vol. 47, no. 11, pp. 1665-1676, 1998.

[42] E. Cerin and E. Leslie, "How socio-economic status contributes to participation in leisure-time physical activity," Social Science \& Medicine, vol. 66, no. 12, pp. 2596-2609, 2008.

[43] K. Ball, A. MacFarlane, D. Crawford, G. Savige, N. Andrianopoulos, and A. Worsley, "Can social cognitive theory constructs explain socio-economic variations in adolescent eating behaviours? A mediation analysis," Health Education Research, vol. 24, no. 3, pp. 496-506, 2009.

[44] C. B. M. Kamphuis, F. J. van Lenthe, K. Giskes, M. Huisman, J. Brug, and J. P. Mackenbach, "Socioeconomic differences in lack of recreational walking among older adults: the role of neighborhood and individual factors," International Journal of Behavioral Nutrition and Physical Activity, vol. 6, no. 1, 2009.

[45] M. S. Businelle, D. E. Kendzor, L. R. Reitzel et al., "Mechanisms linking socioeconomic status to smoking cessation: a structural equation modeling approach," Health Psychology, vol. 29, no. 3, pp. 262-273, 2010.

[46] H. Sugisawa, T. Nomura, and M. Tomonaga, "Psychosocial mediators between socioeconomic status and dietary habits among Japanese older adults," The Journal of Nutrition, Health \& Aging, vol. 19, no. 2, pp. 130-136, 2015.

[47] S. Geyer, Ö. Hemström, R. Peter, and D. Vågerö, "Education, income, and occupational class cannot be used interchangeably in social epidemiology. Empirical evidence against a common practice," Journal of Epidemiology and Community Health, vol. 60, no. 9, pp. 804-810, 2006.

[48] Japan Association of Kidney Disease Patients, “2011 Nendo Ketsuekitosekikanja Jittaichosa Hokokusho (2011 Report on Survey Hemodialysis Patients)," Association of Periodical Publication by Organizations for the Disabled, 2011.

[49] J. M. Cundiff, B. N. Uchino, T. W. Smith, and W. Birmingham, "Socioeconomic status and health: education and income are independent and joint predictors of ambulatory blood pressure," Journal of Behavioral Medicine, vol. 38, no. 1, pp. 9-16, 2013.

[50] S. Clark, K. Farrington, and J. Chilcot, "Nonadherence in dialysis patients: prevalence, measurement, outcome, and psychological determinants," Seminars in Dialysis, vol. 27, no. 1, pp. 42-49, 2014.

[51] Y. Shimizu and H. Sugisawa, "The influences of family support on older patients self-care behaviors of dietary restrictions due to hemodialysis treatment: differences on perception between patients and their family members," Journal of Japan Academy of Home Care, vol. 18, no. 1, pp. 42-50, 2014 (Japanese).

[52] K. Kawabata, Y. Ishida, and M. Oka, "Factors influencing management behavior and self-efficacy in hemodialysis patients," Japanese Journal of Physiological Anthropology, vol. 3, no. 3, pp. 89-96, 1998 (Japanese).

[53] R. Saran, J. L. Bragg-Gresham, H. C. Rayner et al., "Nonadherence in hemodialysis: associations with mortality, hospitalization, and practice patterns in the DOPPS," Kidney International, vol. 64, no. 1, pp. 254-262, 2003. 
[54] A. A. Yusuf, Y. Hu, B. Singh, J. A. Menoyo, and J. B. Wetmore, "Serum potassium levels and mortality in hemodialysis patients: a retrospective cohort study," American Journal of Nephrology, vol. 44, no. 3, pp. 179-186, 2016.

[55] National Kidney Foundation, "K/DOQI clinical practice guidelines for bone metabolism and diseases in chronic kidney Disease," American Journal of Kidney Diseases, vol. 42, Supplement 3, no. 4, pp. 1-201, 2003.

[56] Y. Hou, X. Li, L. Sun, Z. Qu, L. Jiang, and Y. Du, "Phosphorus and mortality risk in end-stage renal disease: A meta-analysis," Clinica Chimica Acta, vol. 474, pp. 108-113, 2017.

[57] S. Baraz, S. Parvardeh, E. Mohammadi, and B. Broumand, "Dietary and fluid compliance: An educational intervention for patients having haemodialysis," Journal of Advanced Nursing, vol. 66, no. 1, pp. 60-68, 2010.

[58] Y. M. Chan, M. S. Zalilah, and S. Z. Hii, "Determinants of compliance behaviours among patients undergoing hemodialysis in malaysia," PLoS ONE, vol. 7, no. 8, Article ID e41362, 2012.

[59] K. J. Preacher and A. F. Hayes, "Asymptotic and resampling strategies for assessing and comparing indirect effects in multiple mediator models," Behavior Research Methods, vol. 40, no. 3, pp. 879-891, 2008.

[60] H. Sugisawa, K. Harada, Y. Sugihara, S. Yanagisawa, and M. Shinmei, "Health, psychological, social and environmental mediators between socioeconomic inequalities and participation in exercise among elderly Japanese," Ageing and Society, pp. 1-19, 2019.

[61] L. K. Muthén and B. O. Muthén, Mplus statistical analysis with latent variables: user's guide, Muthén \& Muthén, 1997.

[62] M. W. Browne and R. Cudeck, "Alternative ways of assessing model fit," Sociological Methods \& Research, vol. 21, no. 2, pp. 230-258, 1992.

[63] P. M. Bentler, "Comparative fit indexes in structural models," Psychological Bulletin, vol. 107, no. 2, pp. 238-246, 1990.

[64] C. Kugler, I. Maeding, and C. L. Russell, "Non-adherence in patients on chronic hemodialysis: An international comparison study," Journal of Nephrology, vol. 24, no. 3, pp. 366-375, 2011.

[65] H. Boeing, A. Bechthold, A. Bub et al., "Critical review: vegetables and fruit in the prevention of chronic diseases," European Journal of Nutrition, vol. 51, no. 6, pp. 637-663, 2012.

[66] E. Volpi, W. W. Campbell, J. T. Dwyer et al., "Is the optimal level of protein intake for older adults greater than the recommended dietary allowance?" The Journals of Gerontology, Series A: Biological Sciences and Medical Sciences, vol. 68, no. 6, pp. 677681, 2013.

[67] K. M. Appleton, "Barriers to and facilitators of the consumption of animal-based protein-rich foods in older adults," Nutrients, vol. 8, no. 4, article no 187, 2016.

[68] K. Giskes, M. Avendaňo, J. Brug, and A. E. Kunst, "A systematic review of studies on socioeconomic inequalities in dietary intakes associated with weight gain and overweight/obesity conducted among European adults," Obesity Reviews, vol. 11, no. 6, pp. 413-429, 2010.

[69] N. D. Riediger and M. H. Moghadasian, "Patterns of fruit and vegetable consumption and the influence of sex, age and sociodemographic factors among the Canadian elderly," Journal of the American College of Nutrition, vol. 27, no. 2, pp. 306-313, 2008.

[70] A. Drewnowski and N. Darmon, "Food choices and diet costs: An economic analysis," Journal of Nutrition, vol. 135, no. 4, pp. 900-904, 2005.
[71] S. Bowman, "Low economic status is associated with suboptimal intakes of nutritious foods by adults in the National Health and Nutrition Examination Survey 1999-2002," Nutrition Research, vol. 27, no. 9, pp. 515-523, 2007.

[72] K. M. Jetter and D. L. Cassady, "The availability and cost of healthier food alternatives," American Journal of Preventive Medicine, vol. 30, no. 1, pp. 38-44, 2006.

[73] S. Ghimire, R. L. Castelino, N. M. Lioufas, G. M. Peterson, and S. T. R. Zaidi, "Nonadherence to medication therapy in haemodialysis patients: A systematic review," PLoS ONE, vol. 10, no. 12, Article ID 0144119, 2015.

[74] K. Avlund, B. E. Holstein, M. Osler, M. T. Damsgaard, P. HolmPedersen, and N. K. Rasmussen, "Social position and health in old age: The relevance of different indicators of social position," Scandinavian Journal of Public Health, vol. 31, no. 2, pp. 126-136, 2003.

[75] P. Herd, B. Goesling, and J. S. House, "Socioeconomic position and health: The differential effects of education versus income on the onset versus progression of health problems," Journal of Health and Social Behavior, vol. 48, no. 3, pp. 223-238, 2007.

[76] J. S. House, J. M. Lepkowski, A. M. Kinney, R. P. Mero, R. C. Kessler, and A. R. Herzog, "The social stratification of aging and health," Journal of Health and Social Behavior, vol. 35, no. 3, pp. 213-234, 1994.

[77] J. R. Hébert, "Social desirability trait: biaser or driver of selfreported dietary intake?" Journal of the Academy of Nutrition and Dietetics, vol. 116, no. 12, pp. 1895-1898, 2016. 


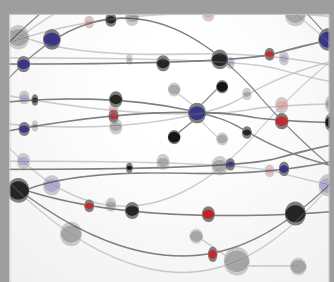

The Scientific World Journal
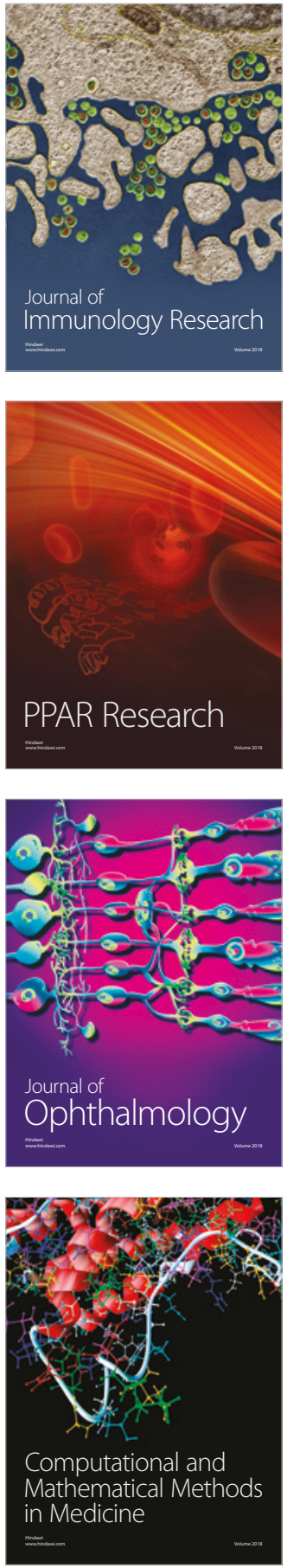

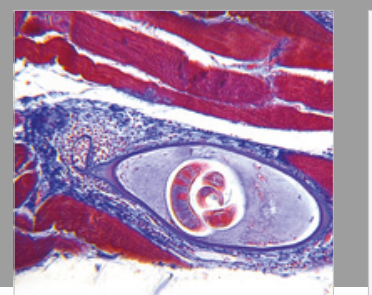

Gastroenterology Research and Practice

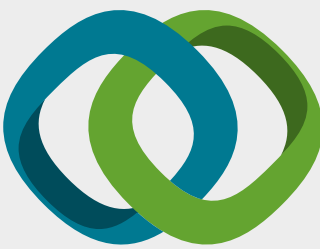

\section{Hindawi}

Submit your manuscripts at

www.hindawi.com
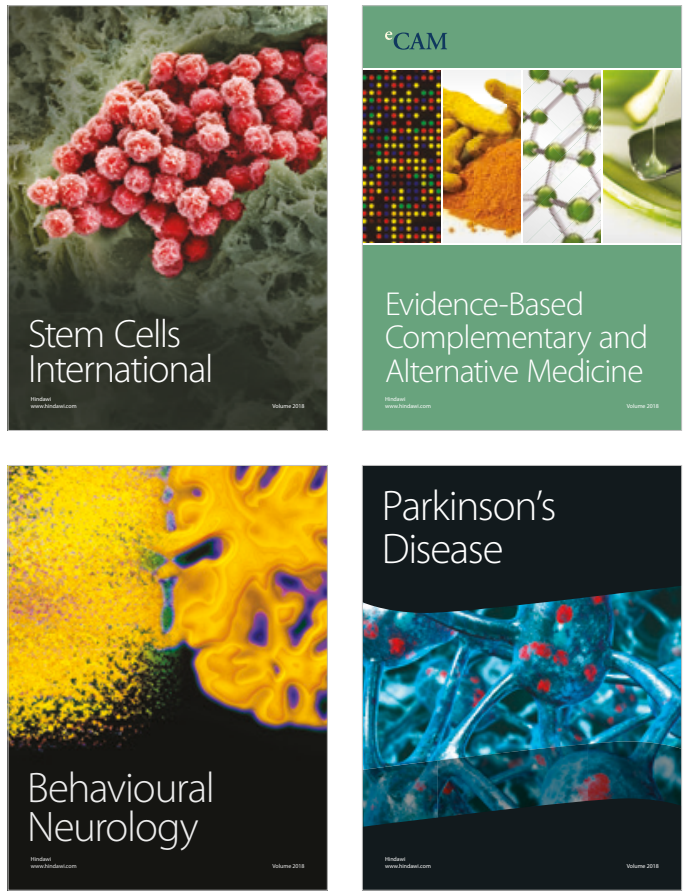

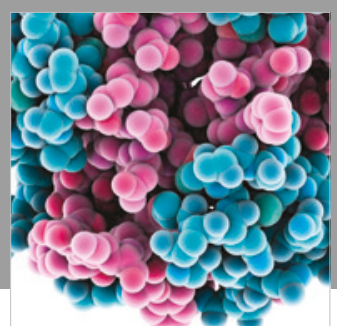

ournal of

Diabetes Research

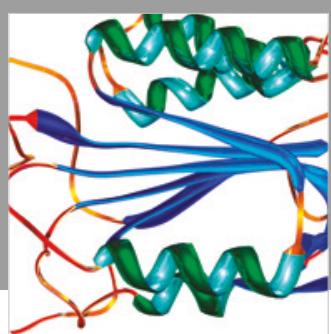

Disease Markers
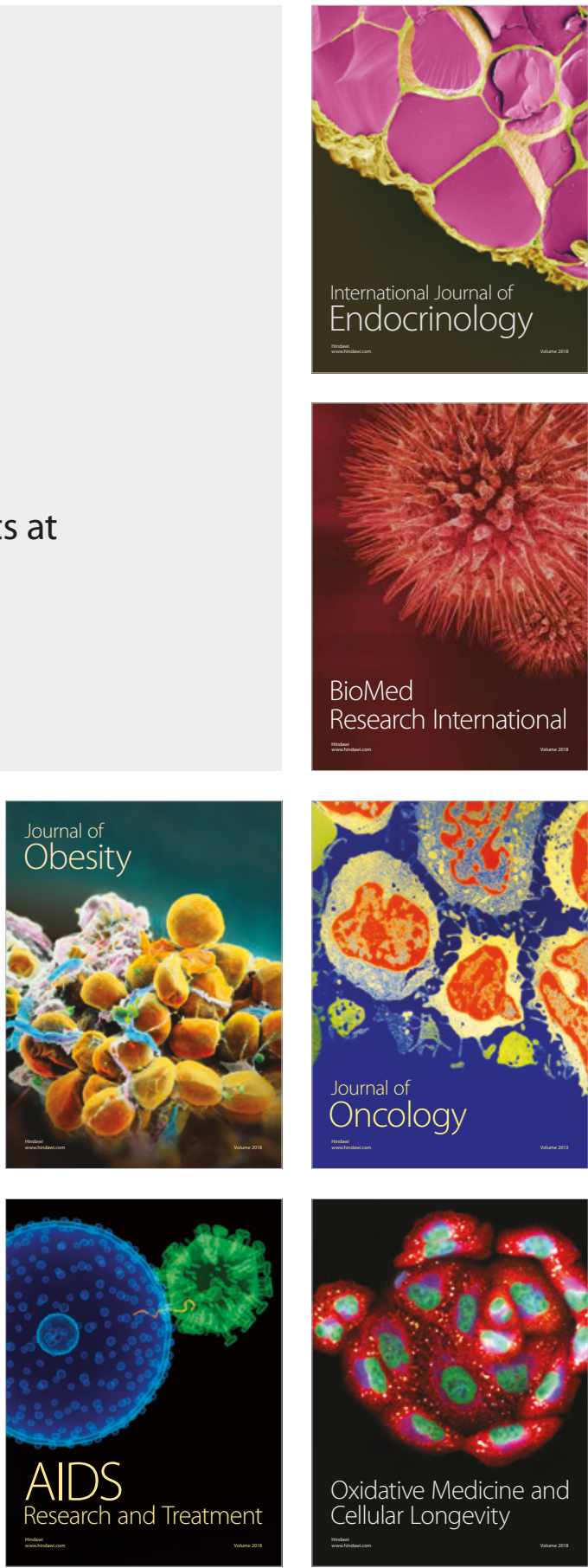